\title{
Development of a Novel Risk Score to Select the Optimal Candidate for Cytoreductive Nephrectomy Among Patients with Metastatic Renal Cell Carcinoma. Results from a Multi-institutional Registry (REMARCC)
}

\author{
Michele Marchioni ${ }^{a, b}$, Maximilian Kriegmair ${ }^{c}$, Mathias Heck ${ }^{d}$, Thomas Amiel ${ }^{d}$, \\ Francesco Porpiglia $^{e}$, Enrico Ceccucci ${ }^{e}$, Riccardo Campi $^{f}$, Andrea Minervini $^{f}$, Andrea Mari $^{f}$, \\ Siska Van Bruwaene ${ }^{g}$, Estefania Linares $^{h}$, Vital Hevia $^{i}$, Mireia Musquera $^{j}$, Mauricio D'Anna $^{j}$, \\ Ithaar Derweesh $^{k}$, Aaron Bradshaw ${ }^{k}$, Riccardo Autorino ${ }^{l}$, Georgi Guruli ${ }^{l}$, Alessandro Veccia $^{l}$, \\ Eduard Roussel $^{m}$, Maarten Albersen ${ }^{m}$, Nicola Pavan ${ }^{n}$, Francesco Claps $^{n}$, Alessandro Antonelli ${ }^{\circ}$, \\ Carlotta Palumbo ${ }^{o}$, Tobias Klatte ${ }^{p}$, Selcuk Erdem ${ }^{q}$, Maria Carmen Mir ${ }^{r, *}$, \\ in collaboration with EAU-Young Academic Urologist Kidney Cancer Group
}

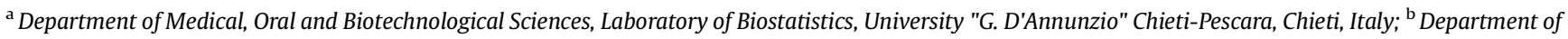
Urology, SS Annunziata Hospital, "G. D'Annunzio" University of Chieti, Chieti, Italy; ' Department of Urology, University Medical Centre Mannheim, Mannheim, Germany; ${ }^{\mathrm{d}}$ Department of Urology, Technical University of Munich, Munich, Germany; ${ }^{\mathrm{e}}$ Department of Urology, School of Medicine, University of Turin-San Luigi Gonzaga Hospital, Turin, Italy; ${ }^{\mathrm{f}}$ Department of Urology, University of Florence, Careggi Hospital, Florence, Italy; ${ }^{\mathrm{g}}$ Department of Urology, AZ Groeninge, Kortrijk,

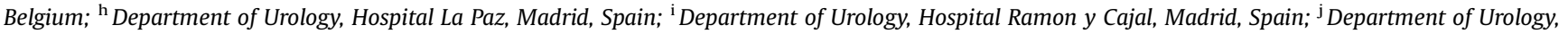
Hospital Clinic, Barcelona, Spain; ${ }^{\mathrm{k}}$ Department of Urology, UC San Diego Moores Cancer Center, University of California San Diego School of Medicine, Louisiana Jolla, CA, USA; ${ }^{1}$ Division of Urology, VCU Medical Center, Richmond, VA, USA; ${ }^{\mathrm{m}}$ Department of Urology, University Hospitals Leuven, Leuven, Belgium; ${ }^{\mathrm{n}}$ Urology Clinic, Department of Medical, Surgical and Health Science, University of Trieste, Trieste, Italy; ${ }^{\circ}$ Department of Urology, University of Verona, AOUI Verona Hospital, Verona, Italy; ${ }^{\mathrm{p}}$ Department of Urology, Royal Bournemouth Hospital, University Hospitals Dorset, Bournemouth, UK; ${ }^{\mathrm{q}}$ Department of Urology, Faculty of Medicine, Istanbul University, Istanbul, Turkey; ${ }^{\mathrm{r}}$ Department of Urology, Fundacion Instituto Valenciano Oncologia, Valencia, Spain

Article info

Article history:

Received 21 September 2020

Received in revised form

17 November 2020

Accepted December 9, 2020

Associate Editor:

Gianluca Giannarini

Keywords:

Renal neoplasm

Total nephrectomy

Metastasis

Prognostic score

\begin{abstract}
Background: Selection of patients for upfront cytoreductive nephrectomy (CN) in metastatic renal cell carcinoma (mRCC) has to be improved.

Objective: To evaluate a new scoring system for the prediction of overall mortality $(\mathrm{OM})$ in mRCC patients undergoing $\mathrm{CN}$.

Design, setting, and participants: We identified a total of 519 patients with synchronous mRCC undergoing CN between 2005 and 2019 from a multi-institutional registry (Registry for Metastatic RCC [REMARCC]).

Outcome measurements and statistical analysis: Cox proportional hazard regression was used to test the main predictors of OM. Restricted mean survival time was estimated as a measure of the average overall survival time up to 36 mo of followup. The concordance index (C-index) was used to determine the model's discrimination. Decision curve analyses were used to compare the net benefit from the REMARCC model with International mRCC Database Consortium (IMDC) or Memorial Sloan Kettering Cancer Center (MSKCC) risk scores.

* Corresponding author. Department of Urology, Fundacion Instituto Valenciano de Oncologia, Valencia, Spain. Tel. +34664454416.

E-mail address: mirmare@yahoo.es (M.C. Mir).
\end{abstract}

https://doi.org/10.1016/j.euo.2020.12.010

2588-9311/@ 2020 Published by Elsevier B.V. on behalf of European Association of Urology. 
Results and limitations: The median follow-up period was 18 mo (interquartile range: 5.9-39.7). Our models showed lower mortality rates in obese patients ( $p=$ $0.007)$. Higher OM rates were recorded in those with bone $(p=0.010)$, liver $(p=$ $0.002)$, and lung metastases $(p<0.001)$. Those with poor performance status $(<80 \%)$ and those with more than three metastases had also higher OM rates $(p=$ 0.026 and 0.040 , respectively). The $\mathrm{C}$-index of the REMARCC model was higher than that of the MSKCC and IMDC models (66.4\% vs $60.4 \%$ vs $60.3 \%$ ). After stratification, 113 (22.0\%) patients were classified to have a favorable (no risk factors), $202(39.5 \%)$ an intermediate (one or two risk factors), and 197 (38.5\%) a poor (more than two risk factors) prognosis. Moreover, $72(17.2 \%)$ and 51 (13.9\%) patients classified as having an intermediate and a poor prognosis according to MSKCC and IMDC categories, respectively, would be reclassified as having a good prognosis according to the REMARCC score.

Conclusions: Our findings confirm the relevance of tumor and patient features for the risk stratification of mRCC patients and clinical decision-making regarding CN. Further prospective external validations are required for the scoring system proposed herein.

Patient summary: Current stratification systems for selecting patients for kidney removal when metastatic disease is shown are controversial. We suggest a system that includes tumor and patient features besides the systems already in use, which are based on blood tests.

(c) 2020 Published by Elsevier B.V. on behalf of European Association of Urology.

\section{Introduction}

Cytoreductive nephrectomy (CN) has been the gold standard treatment for upfront metastatic renal cell carcinoma (mRCC) based on survival outcomes reported prior to the advent of tyrosine kinase inhibitors (TKIs) [1]. The role and the timing of $\mathrm{CN}$ in mRCC have been investigated with inconsistent results. Contrary to retrospective data [2], phase 3 prospective CARMENA clinical trial demonstrated that targeted therapy with sunitinib alone was noninferior to $\mathrm{CN}$ followed by sunitinib in International mRCC Database Consortium (IMDC) intermediate- and poor-risk patients with clear cell histology [3]. SURTIME (immediate vs delayed $\mathrm{CN}$ in synchronic mRCC) results were released with similar answers [4].

To date, $\mathrm{CN}$ is recommended in patients with a good prognosis and in selected intermediate-risk patients $[5,6]$. Patient stratification rely mainly on two risk scores, Memorial Sloan Kettering Cancer Center (MSKCC) and IMDC criteria. These two risk stratification systems have classified mRCC patients into prognostic categories based on a composite of patient laboratory and clinical features (favorable-, intermediate-, and poor-risk categories). Unfortunately, no disease- or pathology-specific features, such as metastatic burden or site, are considered in these two scores.

In the current study, we aimed to test the effect of patient and tumor predictors on overall mortality (OM) in patients undergoing upfront $\mathrm{CN}$ for mRCC. Moreover, we aimed to compare the model developed in our cohort with current scoring systems for the prediction of OM.

\section{Patients and methods}

\subsection{Patient population}

A retrospective review of patients diagnosed with mRCC was abstracted from the Registry for Metastatic RCC (REMARCC) database. The REMARCC collected data on patients who underwent $\mathrm{CN}$ between 2005 and 2019 from 14 North American and European institutions [7]. Institutional Board approval was obtained at all participating institutions when required. Patients referred with renal cell carcinoma (RCC) underwent initial staging evaluation including cross-sectional imaging of chest and abdomen/pelvis with additional studies as indicated [6]. Decision to proceed with $\mathrm{CN}$, systemic therapy, and/or metastasectomy was conducted at the discretion of the treating team. Type of surgery and surgical approach were determined according to surgeon discretion. Type of systemic therapy and treatment protocols were determined on an institutional level. Radiographic follow-up and determination of response were conducted by the Response Evaluation Criteria in Solid Tumors [8].

\subsection{Data collection}

Patients were subdivided into those with favorable (MSKCC score 0), intermediate (MSKCC score 1-2), and poor (MSKCC score $\geq 3$ ) prognoses by calculating MSKCC risk scores (time from diagnosis to systemic treatment, hemoglobin [HGB] concentration below the lower limit of normal [defined as $13.5 \mathrm{~g} / \mathrm{dl}$ for men and $12.0 \mathrm{~g} / \mathrm{dl}$ for women], calcium $>10 \mathrm{mg} / \mathrm{dl}$, lactic dehydrogenase [LDH] $>1.5$ times the upper limit of normal [defined as $140 \mathrm{U} / \mathrm{l}$ ], and Karnofsky performance status $<80 \%$ ) [9]. Multiple studies have provided external validation for the MSKCC score accurately predicting median survival times in patients with mRCC [10].

IMDC risk stratification was calculated, including time from diagnosis to systemic treatment $<1 \mathrm{yr}$, HGB concentration below the lower limit of normal, corrected calcium, neutrophils above the upper limit of normal 
$\left(7 \times 10^{9} / 1\right)$, platelets above the upper limit of normal (400 000 cells $\left./ \mu \mathrm{l}\right)$, and Karnofsky performance status $<80 \%$. Based on IMDC risk scores, patients were also stratified into those having favorable (IMDC score 0 ), intermediate (IMDC score $1-2$ ), and poor (IMDC score $\geq 3$ ) prognoses [10]. Individual scores were calculated by each institution.

All patients with data about the vital status and the follow-up were included. Only patients who underwent upfront CN after 2005 were included in the analysis (considering that date as accepted initiation of targeted therapies).

\subsection{Covariates of interest}

Data were entered into institutional datasets by database managers. The main demographic and clinical characteristics considered for this study were as follows: gender, body mass index (normal weight [18.5-25], overweight [25.01-30.0], and obese [>30.0]), Eastern Cooperative Oncology Group performance status, Karnofsky performance status ( $<80 \%$ vs $\geq 80 \%$ ) [11], MSKCC risk categories, IMDC risk categories, and time from diagnosis to medical therapy. The year of surgery was further stratified (2005-2015 vs 2016-2019) since nivolumab was first introduced as an immunotherapy drug for mRCC in 2015 [12]. Laboratory parameters taken into account were LDH, HGB, calcium, platelet count, lymphocyte count, and neutrophil count. The neutrophil to lymphocyte ratio was also estimated.

The tumor-related characteristics abstracted were clinical and pathological $\mathrm{T}$ stage, clinical and pathological $\mathrm{N}$ stage, primary tumor histology, presence of sarcomatoid dedifferentiation within the primary tumor, pathological tumor size, number of metastases at diagnosis (defined as the number of lesions at imaging), and site of metastases.

\subsection{Statistical analyses}

The primary outcome of the current study was OM measured from the date of diagnosis to the date of last follow-up. Descriptive statistics relied on median and interquartile ranges (IQRs) for continuous covariates, and on absolute and relative frequencies for categorical covariates. Several analytic steps were carried out. First, uni- and multivariable Cox proportional hazard regression models were performed to test the main predictors of OM. Covariates included in the multivariable models were those statistically significant at the univariable analyses. Second, we tested the proportional hazard assumption for the overall multivariable model and for each of the included covariates with the Grambsch and Therneau [13] test. Third, the restricted mean survival time (RMST) was estimated as a measure of the average overall survival time up to 36 mo of follow-up. The RMST is equivalent to the area under the Kaplan-Meier curve and is based on all patients' exposure times. When the proportional hazard assumption is valid, the RMST is as powerful as the log-rank test and even more powerful if the assumption is violated [14]. The concordance index (C-index) and its 95\% confidence interval (CI) were estimated and internally validated based on 300 bootstraps as a measure of the 36-mo OM discriminative power of the model. Fourth, decision curve analyses (DCAs) were used to compare the net benefit derived from the use of our model with that from IMDC/MSKCC categories (Fig. 1) [7]. Fifth, our population was stratified according to the number of risk factors harbored by each patient in three subgroups, namely, those with favorable (no risk factors), intermediate (one or two risk factors), or poor (more than two risk factors) prognosis. Finally, to explore the survival advantage of those reclassified as having favorable risk according to our score, a sensitivity analysis was performed estimating the RMST difference within the subgroup of patients with an intermediate or a poor prognosis according to IMDC/MSKCC categories.

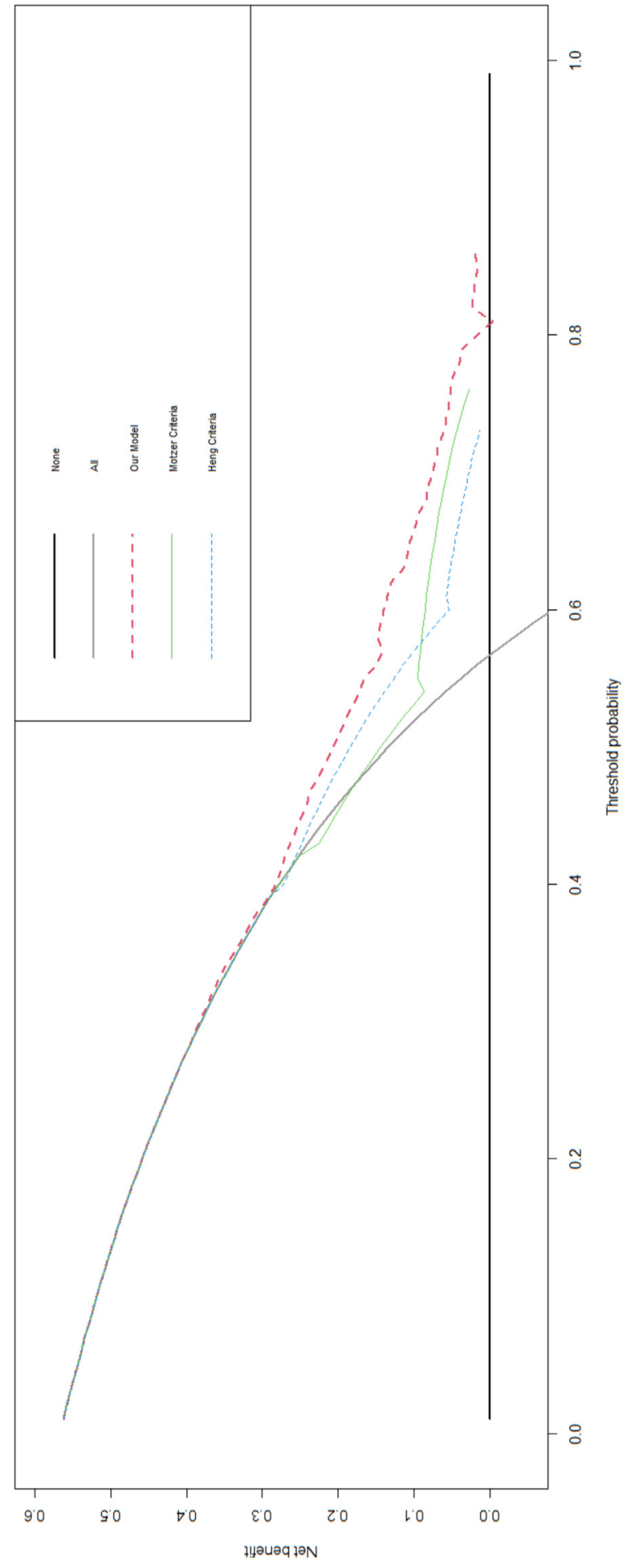

Fig. 1 - Decision curve analyses graphically depicting the net benefit derived from the proposed multivariable Cox regression model, including BMI classes, site of metastases (bone, liver, and lung), number of metastases, and performance status compared with the IMDC and MSKCC scores. BMI = body mass index; IMDC = International mRCC Database Consortium; $\mathbf{m R C C}=$ metastatic renal cell carcinoma; MSKCC = Memorial Sloan Kettering Cancer Center. 
All statistical analyses were performed using R statistical software (version 4.0.0; R Foundation for Statistical Computing, Vienna, Austria). All tests were two tailed, and $p<0.05$ was considered statistically significant.

\section{Results}

\subsection{Clinical and pathological characteristics of the cohort analyzed}

Overall, 519 patients were included in the analyses (Table 1). Most of the included patients were male (71.3\%) and $27.7 \%$ were obese. The median age at diagnosis was 63.0 (IQR: 55.0-70.2) yr. The vast majority of patients received $\mathrm{CN}$ between 2005 and 2015 (79.8\%). Most of the patients were classified to have intermediate risk according to MSKCC (63.6\%) and IMDC (55.5\%) criteria. The median time from diagnosis to medical therapy was 4.0 (IQR: 2.5-30.0) mo. The median time from $\mathrm{CN}$ to systemic treatment was 28.0 (IQR: 14.0-49.0) d. After surgery, most of the patients were classified as pT3-4 (73.6\%) and pN1 (52\%). Most of the patients had clear-cell RCC histology (65.5\%), while sarcomatoid dedifferentiation was found in $20.8 \%$ of all patients. The most frequent site of metastases was lung (52.6\%). Notably, $30.4 \%$ of all patients harbored more than three metastatic sites at diagnosis.

\subsection{Clinical predictors of OM in cohort of patients with mRCC}

During a median follow-up of 18.1 (IQR: 5.9-39.7) mo, 296 (57.8\%) deaths were recorded. The median overall survival was 27.8 (95\% CI: 23.5-30.9) mo. The Grambsch and Therneau [13] test failed to confirm a violation of the proportional hazard assumption for the multivariable Cox regression model investigating principal OM predictors $(p=$ 0.637). Our models showed lower mortality rates in obese patients than in their normal-weight counterparts (hazard ratio [HR]: $0.56,95 \% \mathrm{CI}$ : $0.37-0.86, p=0.007$; Table 2). Moreover, RMST analyses showed that obese patients had $3.95 \mathrm{mo}$ (95\% CI: $0.77,7.14 \mathrm{mo}, p=0.015)$ of overall survival gain at 36 mo compared with their nonobese counterparts (Table 3).

Our models confirmed that the sites of metastases have an effect on OM. In particular, patients with bone (HR: 1.49, 95\% CI: 1.10-2.03, $p=0.010$ ), liver (HR: $1.71,95 \% \mathrm{CI}: 1.25-$ $2.62, p=0.002)$, and lung (HR = 1.60, 95\% CI: 1.26-2.04, $p<$ 0.001 ) metastases had increased OM. Conversely, Cox regression models failed to show any effect of brain and mediastinal node metastases (Table 2). Furthermore, RMST analyses showed that patients with liver metastases had the largest survival loss at $36 \mathrm{mo}(-8.25 \mathrm{mo}, p<0.001$; Table 3).

Among patient characteristics, having a performance status of $<80 \%$ was associated with increased OM (HR: 1.50 , 95\% CI: $1.05-2.14, p=0.026)$; however, RMST showed that this disadvantage in terms of survival time was statistically significant at $24 \mathrm{mo}(-2.82 \mathrm{mo}, p=0.036)$, but not at $36 \mathrm{mo}$ $(-3.76 \mathrm{mo}, p=0.081)$.
Table 1 - Main demographic and clinical characteristics at baseline of patients diagnosed with metastatic renal cell carcinoma treated with cytoreductive nephrectomy

\begin{tabular}{|c|c|}
\hline & Overall $(N=519)$ \\
\hline Age at diagnosis (yr) & $63.0(55-70)$ \\
\hline \multicolumn{2}{|l|}{ Year of surgery, $n(\%)$} \\
\hline $2005-2015$ & $414(79.8)$ \\
\hline 2016-2019 & $105(20.2)$ \\
\hline Male, $n(\%)$ & $370(71)$ \\
\hline BMI $\left(\mathrm{mg} / \mathrm{m}^{2}\right)$ & $26.0(24-29)$ \\
\hline \multicolumn{2}{|l|}{ BMI classification, $n$ (\%) } \\
\hline Normal & $144(28)$ \\
\hline Obese & $78(15)$ \\
\hline Overweight & $145(28)$ \\
\hline Unknown & $152(29)$ \\
\hline ECOG PS > $2, n(\%)$ & $118(28)$ \\
\hline Performance status $<80 \%, n(\%)$ & $61(12)$ \\
\hline LDH (UI/I) & $250.0(178-322)$ \\
\hline $\mathrm{HGB}(\mathrm{g} / \mathrm{dl})$ & $12.5(11-14)$ \\
\hline Calcium (mg/dl) & $8.7(2.3-9.5)$ \\
\hline Platelet (cells $\left.\times 10^{3} / \mu \mathrm{l}\right)$ & $295.0(215-379)$ \\
\hline Neutrophil (cells $\times 10^{9} / \mathrm{l}$ ) & $5.5(4.4-7.4)$ \\
\hline LDH >1.5 ULN, $n(\%)$ & $230(44)$ \\
\hline $\mathrm{NLR} \geq 4, n(\%)$ & $83(32)$ \\
\hline Time from diagnosis to medical therapy (mo) & $4.0(2.5-30.0)$ \\
\hline \multicolumn{2}{|l|}{ Motzer risk stratification, $n$ (\%) } \\
\hline Favorable & $100(19)$ \\
\hline Intermediate & $330(64)$ \\
\hline Poor & $89(17)$ \\
\hline \multicolumn{2}{|l|}{ Heng risk stratification, $n(\%)$} \\
\hline Favorable & $151(29)$ \\
\hline Intermediate & $288(55)$ \\
\hline Poor & $80(15)$ \\
\hline Deaths during follow-up, $n$ (\%) & $306(59)$ \\
\hline \multicolumn{2}{|l|}{ Clinical T stage, $n(\%)$} \\
\hline cT1-3 & $452(87)$ \\
\hline cT4 & $50(10)$ \\
\hline cTx & $17(3)$ \\
\hline \multicolumn{2}{|l|}{ Clinical $N$ stage, $n(\%)$} \\
\hline $\mathrm{cNO}$ & $237(46)$ \\
\hline $\mathrm{cN} 1$ & $268(52)$ \\
\hline $\mathrm{cNx}$ & $14(3)$ \\
\hline \multicolumn{2}{|l|}{ Pathological T stage, $n(\%)$} \\
\hline pT1-2 & $109(21)$ \\
\hline pT3-4 & $382(73)$ \\
\hline pTx & $28(5)$ \\
\hline \multicolumn{2}{|l|}{ Pathological N stage, $n$ (\%) } \\
\hline pNO & $193(37)$ \\
\hline pN1 & $273(53)$ \\
\hline $\mathrm{pNx}$ & $53(10)$ \\
\hline \multicolumn{2}{|l|}{ Primary tumor histology, $n$ (\%) } \\
\hline ccRCC & $340(65)$ \\
\hline Non-ccRCC & $82(16)$ \\
\hline Unknown & $97(19)$ \\
\hline Sarcomatoid dedifferentiation, $n$ (\%) & $89(21)$ \\
\hline Pathological tumor size $(\mathrm{cm})$ & $9(6.8-11.5)$ \\
\hline Number of metastases at diagnosis & $2(1-4)$ \\
\hline$>3$ metastases at diagnosis, $n(\%)$ & $157(30)$ \\
\hline Bone metastases, $n(\%)$ & $74(14)$ \\
\hline Brain metastases, $n(\%)$ & $31(6)$ \\
\hline Liver metastases, $n$ (\%) & $51(10)$ \\
\hline Lung metastases, $n$ (\%) & $273(53)$ \\
\hline Mediastinal lymph nodes metastases, $n$ (\%) & $54(10)$ \\
\hline Largest metastatic site size $(\mathrm{cm})$ & $2.0(1.5-3.5)$ \\
\hline \multicolumn{2}{|c|}{$\begin{array}{l}\text { BMI = body mass index; } c \text { CRCC = clear cell renal cell carcinoma; ECOG PS = } \\
\text { Eastern Cooperative Oncology Group performance status; HGB = } \\
\text { hemoglobin; LDH = lactic dehydrogenase; NLR = neutrophil to } \\
\text { lymphocyte ratio; ULN = upper limit of normal. } \\
\text { Continuous variables are reported as median and interquartile ranges. } \\
\text { Categorical covariates are reported as absolute and relative frequencies (\%). }\end{array}$} \\
\hline
\end{tabular}


Table 2 - Univariable and multivariable Cox proportional hazard models predicting overall mortality

\begin{tabular}{|c|c|c|}
\hline Overall mortality & $\begin{array}{c}\text { Univariable analyses } \\
\text { Hazard radio (95\% CI, } p \text { value) }\end{array}$ & $\begin{array}{c}\text { Multivariable analyses } \\
\text { Hazard radio ( } 95 \% \text { CI, } p \text { value) }\end{array}$ \\
\hline Age at diagnosis & $1.01(0.99-1.02, p=0.167)$ & - \\
\hline Year of surgery (2016-2019 vs $2005-2015$ ) & $0.90(0.63-1.27, p=0.540)$ & - \\
\hline Male vs female & $1.04(0.80-1.35, p=0.764)$ & - \\
\hline \multicolumn{3}{|l|}{ BMI classes } \\
\hline Normal weight & Reference & Reference \\
\hline Obese & $0.50(0.33-0.75, p=0.001)$ & $0.56(0.37-0.86, p=0.007)$ \\
\hline Overweight & $0.83(0.62-1.11, p=0.210)$ & $0.87(0.65-1.17, p=0.353)$ \\
\hline Unknown & $0.82(0.61-1.10, p=0.177)$ & $0.91(0.67-1.24, p=0.550)$ \\
\hline Bone metastases & $1.75(1.31-2.33, p<0.001)$ & $1.49(1.10-2.03, p=0.010)$ \\
\hline Brain metastases & $0.92(0.58-1.45, p=0.723)$ & - \\
\hline Liver metastases & $2.26(1.58-3.23, p<0.001)$ & $1.81(1.25-2.62, p=0.002)$ \\
\hline Lung metastases & $1.66(1.31-2.10, p<0.001)$ & $1.60(1.26-2.04, p<0.001)$ \\
\hline Mediastinal node metastases & $1.27(0.92-1.75, p=0.148)$ & - \\
\hline Number of metastases ( $>3$ vs $\leq 3$ ) & $1.59(1.26-2.00, p<0.001)$ & $1.29(1.01-1.65, p=0.040)$ \\
\hline Performance status $<80 \%$ & $1.45(1.02-2.06, p=0.037)$ & $1.50(1.05-2.14, p=0.026)$ \\
\hline \multicolumn{3}{|l|}{ Clinical T stage } \\
\hline CT1-3 & Reference & Reference \\
\hline cT4 & $1.11(0.75-1.65, p=0.590)$ & - \\
\hline $\mathrm{cNx}$ & $0.85(0.42-1.72, p=0.652)$ & - \\
\hline \multicolumn{3}{|l|}{ Clinical $\mathrm{N}$ stage } \\
\hline cNO & Reference & Reference \\
\hline $\mathrm{cN} 1$ & $1.23(0.97-1.55, p=0.082)$ & - \\
\hline $\mathrm{cNx}$ & $0.94(0.44-2.02, p=0.875)$ & - \\
\hline $\operatorname{NLR}(\geq 4$ vs $<4)$ & $0.95(0.69-1.33, p=0.780)$ & - \\
\hline
\end{tabular}

Table 3 - Delta of restricted mean survival time (RMST) at 24 and 36 mo of follow-up for covariates included in the new score

\begin{tabular}{|c|c|c|c|c|}
\hline & $\begin{array}{c}\Delta \text { RMST at } 24 \text { mo } \\
(95 \% \mathrm{CI})\end{array}$ & $p$ value & $\Delta$ RMST at $36 \mathrm{mo}(95 \% \mathrm{CI})$ & $p$ value \\
\hline Obesity & $2.39(0.51,4.27)$ & 0.013 & $3.95(0.77,7.14)$ & 0.015 \\
\hline Bone metastases & $-2.23(-4.12,-0.05)$ & 0.045 & $-4.66(-7.99,-1.34)$ & 0.006 \\
\hline Liver metastases & $-4.65(-7.55,-1.75)$ & 0.002 & $-8.25(-12.32,-4.18)$ & $<0.001$ \\
\hline Lung metastases & $-2.48(-3.98,-0.97)$ & 0.001 & $-4.24(-6.67,-1.82)$ & 0.001 \\
\hline Number of metastases ( $>3$ vs. $\leq 3$ ) & $-2.55(-4.19,-0.90)$ & 0.002 & $-4.42(-7.01,-1.82)$ & 0.001 \\
\hline Performance status $<80 \%$ & $-2.82(-5.45,-0.18)$ & 0.036 & $-3.76(-7.98,0.47)$ & 0.081 \\
\hline
\end{tabular}

\subsection{Model accuracy: comparison with established MSKCC and IMDC criteria}

Overall our Cox model showed fair accuracy with a C-index of 66.4\% (95\% CI: 61.5-70.5\%), higher than MSKCC (C-index: 60.4\%, 95\% CI: 56.6-64.2\%) and IMDC (C-index: 60.3\%, 95\% CI: 56.0-63.9\%) criteria. DCAs also showed a slight advantage of our model predicting 36-mo OM. The net benefit was superior to all the other strategies up to $80 \%$ of threshold probabilities.

After stratification according to the number of risk factors identified within our model, $22.0 \%$ of patients were classified as having a favorable (no risk factors), 39.5\% an intermediate (one or two risk factors), and $38.5 \%$ a poor (more than two risk factors) prognosis. Those with a poor prognosis had shorter RMST up to 36 mo than those with intermediate ( $\Delta$ RMST: $-4.2 \mathrm{mo}, 95 \% \mathrm{CI}:-6.95$ to $-1.44, p=$ 0.003 ) and favorable ( $\Delta$ RMST: $-7.56 \mathrm{mo}, 95 \% \mathrm{CI}:-10.66$ to $-4.64, p<0.001)$ prognoses. Similarly, those with an intermediate prognosis had shorter RMST up to 36 mo than those with a favorable prognosis ( $\Delta$ RMST: $-3.37 \mathrm{mo}, 95 \% \mathrm{CI}$ : -6.46 to $-0.28, p=0.033$ ).

Among patients classified as having intermediate/poor prognosis according to MSKCC, 72 (17.2\%) would be reclassified as those having a good prognosis according to the REMARCC score (Supplementary Table 1). These patients had a statistically significant survival advantage compared with those reclassified as having a poor prognosis (36-mo $\Delta$ RMST: 7.44 mo, 95\% CI: 3.76-11.12, $p<0.001$ ). This advantage remained apparent even if not statistically significant differences were seen in comparison with intermediate prognosis patients (36-mo $\Delta$ RMST: $3.28 \mathrm{mo}$, 95\% CI: -0.45 to $7.01, p=0.090$ ).

Similarly, among patients classified to have intermediate/poor prognosis according to IMDC categories, 51 (13.9\%) would be reclassified as those having a good prognosis according to the REMARCC score. These patients had a statistically significant survival advantage compared with 
those reclassified as having a poor prognosis (36-mo $\Delta$ RMST: 6.80 mo, 95\% CI: 2.48-11.12, $p=0.002)$. This advantage remained apparent even if not statistically significant differences were seen in comparison with intermediate prognosis patients (36-mo $\Delta$ RMST: $2.54 \mathrm{mo}$, 95\% CI: -1.87 to $6.96, p=0.258)$.

\section{Discussion}

In the current study, we sought to identify clinical factors to improve mRCC patient prognostic stratification for upfront $\mathrm{CN}$. Moreover, we aimed to explore the accuracy of current scoring systems, namely, IMDC and MSKCC categories. We also explored the possible advantage of reclassification based on characteristics not included within the currently available scoring systems; a specific focus on metastatic status was introduced (number and site of metastases). Our results highlight the relevance of metastatic (organ) location as well as the number of metastatic sites (above 3) as important tumor factors. On the contrary, patient factors that provide a difference in survival are obesity and performance status.

Current widely accepted staging scores for synchronic mRCC include mostly laboratory parameters, whereas metastatic tumoral volume or location is included in the analysis. As a result, a patient with a single liver metastasis or a thousand lung metastases belong to the same category as long as they present with determinate laboratory parameters. It is well established that patients who benefit the most from $\mathrm{CN}$ are younger ones with minimal comorbidities and a low metastatic burden [15]. In addition, using different risk features, we were able to reclassify, as at good prognosis, up to $17 \%$ of patients who would be considered as having intermediate/poor risk based on current risk stratification systems. According to the current guidelines [6], these patients should not undergo CN. The latter might represent an undertreatment of a not negligible proportion of patients who might experience a survival benefit derived from $\mathrm{CN}$. In other words, following the IMDC or MSKCC definition of prognostic group might result in undertreating up to $15-20 \%$ of patients considered to have an intermediate or a poor prognosis.

Metastatic location and volume have previously been explored for prognostic evaluation at the time of $\mathrm{CN}$ with controversial outcomes. Small retrospective cohorts evaluating the tumor volume at mRCC diagnosis did not show a correlation with number and prognosis in intermediate IMDC subgroup stratification [16,17]. Conversely, Capitanio et al [18] observed an overall survival advantage in patients with a single metastatic site independently of the organ location. Our results highlight the relevance of tumor burden outside of the kidney (more than three metastatic sites) together with location (liver) in a predictive stratification model for mRCC. An initial analysis from large centers such as MD Anderson, including a total of 566 patients undergoing $\mathrm{CN}$ in the pretargeted therapy era, had already shown the relevance of liver metastasis as a poor predictor of survival outcomes [19]. In a recent update on the MD Anderson analysis of the predictors of poor prognosis in patients with $\mathrm{MRCC}$ receiving $\mathrm{CN}$ in the TKI era, a total of 608 patients were included. Liver metastasis was excluded from the predictors, adding bone metastasis instead [20]. Hematological and biochemical parameters (ie, $\mathrm{LDH}$, neutrophil to lymphocyte ratio $>4$, low albumin, Creactive protein, and cholesterol) have been correlated with shorter survival outcomes. Of note, studies that include each variable as a single entity of interest do not provide a complete analysis [21,22].

On the contrary, two patient factors have shown its relevance as predictors, obesity, and poor performance status. A strong body of evidence supports obesity (and overweight) as a risk factor for RCC incidence. A recent meta-analysis including over 21 cohort studies and 1500 obese patients reported a 1.7 -fold increased incidence of RCC for obese patients. Moreover, a 4\% increase risk of RCC was observed for each increment of $1 \mathrm{~kg} / \mathrm{m}^{2}$ [23]. Conversely, several authors have suggested a protective effect of obesity in terms of survival outcomes [24]. The obesity paradox has been described in all cancer stages. Metastatic kidney cancer patients were analyzed on the IMDC database, setting body mass index over 25 as a threshold for overweight. Obese metastatic patients had an overall survival improvement of 9 mo versus nonobese patients (25 vs $17 \mathrm{mo}$ ) independently of the prognostic group [25].

Preoperative poor performance status is likely to play a role as a predictor, most probably as a surrogate of a weak systemic response. In our analysis, performance status below $80 \%$ did not hold as a predictor of long-term survival. In the current analysis, $12 \%$ of the population undergoing surgery had performance status of $<80 \%$. This mirrors the results of other published data and supports the current clinical practice based on MSKCC and IMDC criteria. The small representative sample may have precluded conclusions on our results. Other authors have reported $30 \%$ of patients undergoing $\mathrm{CN}$ being unable to receive systemic therapy afterward, due to either progression or perioperative morbidity. Kutikov et al [26] studied a mixed cohort of mRCC patients treated between 1997 and 2008 (treated with classic immunotherapy and targeted therapy). They consistently reported symptomatic disease and performance status below $80 \%$ as predictors of risk of death. Perioperative morbidity related to $\mathrm{CN}$ has been described poorly. In our previous report, we have shown the safety of the procedure within the contemporary era. On the recently published REMARCC data registry, the overall complication rate for $\mathrm{CN}$ was $30 \%$, with only $6 \%$ being reported as major complications (grade 3-5 Clavien-Dindo). Less than $12 \%$ of the patients were readmitted within $30 \mathrm{~d}$ of surgery [7].

The current analysis is not devoid of limitations. The retrospective design carries inherent limitations to data collection. Moreover, as a multicenter study, variation in selection criteria introduces confounding variables. Data belong to large academic tertiary referral centers, which might not be applicable to the general practice; however, it is well recognized that treatment of the subset of patients analyzed should be deferred to referral centers. As with any 
retrospective cohort, a selection bias exists, and patients who received upfront systemic therapy and who did not undergo $\mathrm{CN}$ were not included for analysis. In addition, data granularity did not allow us to report in detail the systemic treatment used or the number of treatments received. However, it should be noticed that up to the publication of cornerstone studies from 2016, the use of active surveillance after cytoreduction, deferred $\mathrm{CN}$, and immune checkpoint inhibitors were not standard of care [27]. By contrast, $\mathrm{CN}$ at the time of patient's inclusion was the only strategy providing long-term complete responses in mRCC in some cases. Thus, we can assume that most of included patients received systemic treatment at some point in time. Moreover, our Cox regression model was able to obtain relatively low accuracy (about 66\%), slightly better than previously published scoring systems. However, we acknowledge that ours represents an internal validation process and the reported accuracy might be inflated by the intrinsic limitation of any internal validation process. Nevertheless, the main objective of the current analyses was to identify OM predictors that take into account pathological features of mRCC patients as well. Indeed, to date, no clear indication on metastatic burden or metastatic site can be derived from the available tools. Despite these limitations, these results are representative of a large $\mathrm{CN}$ cohort at high-volume academic centers. Moreover, we relied on RMST to estimate the gain or loss in OM-free survival time between each patient category. RMST showed a statistically significant and clinically meaningful difference in terms of average OM-free survival time up to $7 \mathrm{mo}$ in those reclassified as having a good prognosis according to our criteria. The use of RMST instead of other measures, such as median survival time, allows consideration of outliers also (such as patients who die soon after $\mathrm{CN}$ or those with longer survival) [26].

Our findings call for prospective validation and assessment. Indeed, mRCC management is constantly evolving; primary systemic therapy for mRCC currently includes immunotherapy together with targeted therapy [28]. Thus, we might expect that other more accurate risk factors could be identified in the next future. However, since new systemic treatment regimen (ie, combination regimens) have been introduced since late 2018, data maturity limits the possibility to identify such type of predictors, even in prospective studies. As a consequence, multi-institutional datasets, such as REMARCC, still represent the best source of evidence available, even if referred to TKI era.

\section{Conclusions}

Tumor-related (location and number of metastatic lesions) and patient-related (obesity and performance status) features should be considered when deciding about upfront $\mathrm{CN}$ in mRCC patients. Our newly proposed stratification system is based on these features, and therefore it is easily applicable and reproducible. Moreover, it seems to perform better than traditional scoring systems. Nevertheless, further external validation is needed, ideally in a prospective setting.
Author contributions: Maria Carmen Mir had full access to all the data in the study and takes responsibility for the integrity of the data and the accuracy of the data analysis.

Study concept and design: Mir, Marchioni.

Acquisition of data: Kriegmair, Heck, Amiel, Ceccucci, Daniele Amparore, Campi, Mari, Van Bruwaene, Linares, Hevia, D’Anna, Bradshaw, Guruli, Veccia, Roussel, Claps, Palumbo, Klatte, Erdem.

Analysis and interpretation of data: Marchioni, Mir.

Drafting of the manuscript: Mir, Autorino.

Critical revision of the manuscript for important intellectual content: Kriegmair, Heck, Amiel, Porpiglia, Ceccucci, Daniele Amparore, Campi, Minervini, Mari, Van Bruwaene, Linares, Hevia, Musquera, D’Anna, Derweesh, Bradshaw, Autorino, Guruli, Veccia, Roussel, Albersen, Pavan, Claps, Antonelli, Palumbo, Klatte, Erdem.

Statistical analysis: Marchioni.

Obtaining funding: None.

Administrative, technical, or material support: None.

Supervision: Autorino, Derweesh, Porpiglia, Minervini, Musquera, Guruli, Albersen, Pavan, Antonelli.

Other: None.

Financial disclosures: Maria Carmen Mir certifies that all conflicts of interest, including specific financial interests and relationships and affiliations relevant to the subject matter or materials discussed in the manuscript (eg, employment/affiliation, grants or funding, consultancies, honoraria, stock ownership or options, expert testimony, royalties, or patents filed, received, or pending), are the following: None.

\section{Funding/Support and role of the sponsor: None.}

\section{References}

[1] Flanigan RC, Mickisch G, Sylvester R, Tangen C, Van Poppel H, Crawford ED. Cytoreductive nephrectomy in patients with metastatic renal cancer: a combined analysis. J Urol 2004;171:1071-6.

[2] Hanna N, Sun M, Meyer CP, et al. Survival analyses of patients with metastatic renal cancer treated with targeted therapy with or without cytoreductive nephrectomy: a national cancer data base study. J Clin Oncol 2016;34:3267-75.

[3] Mejean A, Thezenas S, Chevreau C, et al. Cytoreductive nephrectomy $(\mathrm{CN})$ in metastatic renal cancer (mRCC): update on CARMENA trial with focus on intermediate IMDC-risk population. J Clin Oncol 2019;37:4508.

[4] Bex A, Mulders P, Jewett M, et al. Comparison of immediate vs deferred cytoreductive nephrectomy in patients with synchronous metastatic renal cell carcinoma receiving sunitinib: the SURTIME randomized clinical trial. JAMA Oncol 2019;5:164-70.

[5] Bhindi B, Graham J, Wells JC, et al. Deferred cytoreductive nephrectomy in patients with newly diagnosed metastatic renal cell carcinoma. Eur Urol 2020;78:615-23.

[6] Ljungberg B, Albiges L, Abu-Ghanem Y, et al. European Association of Urology Guidelines on Renal Cell Carcinoma: The 2019 Update. Eur Urol 2019;75:799-810.

[7] Roussel E, Campi R, Larcher A, et al. Rates and predictors of perioperative complications in cytoreductive nephrectomy: analysis of the Registry for Metastatic Renal Cell Carcinoma. Eur Urol Oncol 2020;3:523-9.

[8] Schwartz LH, Mazumdar M, Wang L, et al. Response assessment classification in patients with advanced renal cell carcinoma treated on clinical trials: effect of measurement criteria and other parameters. Cancer 2003;98:1611-9. 
[9] Motzer RJ, Bacik J, Schwartz LH, et al. Prognostic factors for survival in previously treated patients with metastatic renal cell carcinoma.J Clin Oncol 2004;22:454-63.

[10] Heng DYC, Xie W, Regan MM, et al. External validation and comparison with other models of the International Metastatic RenalCell Carcinoma Database Consortium prognostic model: a population-based study. Lancet Oncol 2013;14:141-8.

[11] Heng DYC, Xie W, Regan MM, et al. Prognostic factors for overall survival in patients with metastatic renal cell carcinoma treated with vascular endothelial growth factor-targeted agents: results from a large, multicenter study. J Clin Oncol 2009;27:5794-9.

[12] Hahn AW, Klaassen Z, Agarwal N, et al. First-line treatment of metastatic renal cell carcinoma: a systematic review and network meta-analysis. Eur Urol Oncol 2019;2:708-15.

[13] Grambsch PM, Therneau TM. Proportional hazards tests and diagnostics based on weighted residuals. Biometrika 1994;81:515-26.

[14] Pak K, Uno H, Kim DH, et al. Interpretability of cancer clinical trial results using restricted mean survival time as an alternative to the hazard ratio. JAMA Oncol 2017;3:1692-6.

[15] Larcher A, Wallis CJD, Bex A, et al. Individualised indications for cytoreductive nephrectomy: which criteria define the optimal candidates? Eur Urol Oncol 2019;2:365-78.

[16] Pierorazio PM, McKiernan JM, McCann TR, Mohile S, Petrylak D, Benson MC. Outcome after cytoreductive nephrectomy for metastatic renal cell carcinoma is predicted by fractional percentage of tumour volume removed. BJU Int 2007;100:755-9.

[17] de Bruijn RE, Nijkamp J, Noe A, et al. Baseline tumor volume in assessing prognosis of patients with intermediate-risk synchronous metastatic renal cell carcinoma. Urol Oncol Semin Orig Investig 2016;34:258.e7-13.

[18] Capitanio U, Abdollah F, Matloob R, et al. Effect of number and location of distant metastases on renal cell carcinoma mortality in candidates for cytoreductive nephrectomy: implications for multimodal therapy. Int J Urol 2013;20:572-9.
[19] Culp SH, Tannir NM, Abel EJ, et al. Can we better select patients with metastatic renal cell carcinoma for cytoreductive nephrectomy? Cancer 2010;116:3378-88.

[20] McIntosh Ag, Umbreit Ec, Holland Lc, et al. Optimizing patient selection for cytoreductive nephrectomy based on outcomes in the contemporary era of systemic therapy. Cancer 2020;126:3950-60.

[21] Baum YS, Patil D, Huang JH, et al. Elevated preoperative neutrophilto-lymphocyte ratio may be associated with decreased overall survival in patients with metastatic clear cell renal cell carcinoma undergoing cytoreductive nephrectomy. Asian J Urol 2016;3:20-5.

[22] Margulis V, Shariat SF, Rapoport Y, et al. Development of accurate models for individualized prediction of survival after cytoreductive nephrectomy for metastatic renal cell carcinoma. Eur Urol 2013;63:947-52.

[23] Wang F, Xu Y. Body mass index and risk of renal cell cancer: a doseresponse meta-analysis of published cohort studies. Int J Cancer 2014;135:1673-86.

[24] Choi Y, Park B, Jeong BC, et al. Body mass index and survival in patients with renal cell carcinoma: a clinical-based cohort and meta-analysis. Int J Cancer 2013;132:625-34.

[25] Albiges L, Ari Hakimi A, Xie W, et al. Body mass index and metastatic renal cell carcinoma: clinical and biological correlations. J Clin Oncol 2016;34:3655-63.

[26] Kutikov A, Uzzo RG, Caraway A, et al. Use of systemic therapy and factors affecting survival for patients undergoing cytoreductive nephrectomy. BJU Int 2010;106:218-23.

[27] Mir MC, Matin SF, Bex A, et al. The role of surgery in the management of metastatic kidney cancer: an evidence-based collaborative review. Minerva Urol Nefrol 2018;70:109-25.

[28] Rini BI, Plimack ER, Stus V, et al. Pembrolizumab plus axitinib versus sunitinib for advanced renal-cell carcinoma. N Engl J Med 2019;380:1116-27. 